\title{
Fls, compétence scolaire et élaboration de ressources didactiques spécifiques
}

\author{
Robert Bouchard \& Chantal Parpette \\ Interactions, Corpus, Apprentissage, Représentations (ICAR) \\ Robert.Bouchard@univ-lyon2.fr
}

\section{Introduction}

Les élèves nouvellement arrivés en France (désormais Ena) bénéficient de fait d'une double exposition linguistique, dans les classes d'accueil d'une part et dans l'environnement de ces classes d'autre part. C'est à dire en particulier dans l'environnement constitué par l'établissement scolaire où ils sont inscrits, et plus particulièrement encore par celui des classes ordinaires qu'ils partagent avec leurs camarades francophones... dès que celles-ci leur sont ouvertes. Ils peuvent donc mettre en œuvre une double appropriation linguistique, associant un apprentissage « littéracié » guidé par l'enseignant spécialisé de la classe d'accueil et une acquisition sociale fondée sur les ressources et les besoins correspondant à leur statut d'élève, comme dans l'enseignement par immersion mis en œuvre au Canada depuis une trentaine d'années. Mais nous avons essayé de montrer ailleurs (Bouchard et Cortier 2007, Bouchard, à paraître) que ce qu'ils doivent développer dans ce double contexte, au delà d'un « français de scolarisation », c'est une " compétence scolaire », c'est à dire la maîtrise des comportements interactionnels, verbaux certes, mais aussi non verbaux, caractéristiques du contexte dans lequel s'opère cette appropriation et que celleci vise en priorité.

C'est à l'optimisation des conditions de cette double appropriation que nous voudrions réfléchir dans cette communication en insistant sur deux axes de travail. Le premier concerne une amélioration de nos connaissances sur le fonctionnement interactionnel des classes normales à la fois site et but de l'acquisition des nouveaux arrivants. Le second doit aboutir à un enrichissement des ressources langagières disponibles dans les classes d'accueil par la constitution d'un matériel ad hoc transposant, didactisant les résultats de la première recherche. Nous laisserons malheureusement de côté une troisième facette de l'action didactique, l'articulation, au sein de dispositifs de formation, de l'action de l'enseignant « de » français et celles des enseignants «en » français, qui ont en charge les mêmes élèves mais nous avons déjà eu l'occasion de l'aborder ailleurs (Cortier 2003, Bouchard et Cortier 2007).

\section{Migration, enseignement des langues et compétence scolaire}

D'un point de vue purement didactique, on peut estimer que, parmi les publics scolaires qui apprennent des langues étrangères, les «Ena » se trouvent dans une situation... enviable. Sur le papier en effet, ils sont ceux qui disposent des meilleures conditions d'appropriation de la langue du pays dans lequel ils sont amenés à vivre. Ils bénéficient d'une double exposition scolaire et non-scolaire à la langue étrangère, qui décuple - tant quantitativement que qualitativement - les ressources linguistiques mises à leur disposition. Cette double exposition leur permet aussi, nous l'avons dit, de cumuler les avantages de l'apprentissage, scolaire, guidé (clases d'accueil) et de l'acquisition sociale (immersion dans la classe normale). D'autre part, du fait même de cette immersion linguistique, ils ressentent les besoins linguistiques et communicatifs indispensables pour mettre en marche les mécanismes acquisitionnels spontanés que l'on peut postuler. La conjonction ressources-besoins est donc optimale : ils ont toutes chances en particulier de mieux s'approprier la langue du pays d'accueil que leurs camarades restés au pays et apprenant cette langue dans le cadre de la seule classe de langue.

Bien sûr, quand on prend en compte non seulement sa situation d'appropriation de la langue mais la finalité et les caractéristiques temporelles de celle-ci, la situation de l'Ena apparait, moins idéale, plus soumise à de fortes contraintes. 
Ainsi, alors que l'apprentissage guidé des langues est la plupart du temps lent et progressif, l'Ena est soumis, quant à lui, à la nécessité d'un apprentissage rapide de cette langue dans la mesure où, de la qualité de cet apprentissage dépend l'ensemble de ses autres apprentissages comme une socialisation satisfaisante dans son nouveau cadre de vie scolaire. Mais surtout, dans ce court délai, il est sommé de réaliser - exigence exceptionnelle en didactique des langues - un apprentissage « rattrapant » celui des natifs, et aboutissant à une compétence communicative, orale et écrite, égale à la leur. Il doit développer un bilinguisme strictement additif, sans bénéficier de la connivence d'une communauté bilingue et des possibilités du code-switching et des «parlers bilingues ». Autant qu'un bilinguisme, il doit manifester une double compétence linguistique et culturelle qui lui permette de se comporter globalement en classe selon les attentes implicites du reste de la communauté scolaire dont il doit devenir partie prenante. Un Ena « intégré» à l'école - l'institution sociale où il est amené à passer la plus grande partie de son temps et au-delà tout élève « heureux » à l'école - ne peut être qu'un élève qui accepte de « se fondre » - au moins momentanément - dans la communauté de la classe dont il a réussi à reconstituer l' « habitus » (cf Lahire 2001, 2006, pour une discussion sur le concept proposé par Bourdieu). Si l'école est un lieu d'apprentissage, c'est aussi une institution éducative qui a pour but de construire implicitement des sujets, partageant un ensemble de pratiques sinon de valeurs... ayant trait en particulier à la vie en commun entre enfants/adolescents comme entre enfants/adolescents et adulte(s). Pour alléger notre propos, affirmons cependant, toujours avec B. Lahire (2001), que l'homme est «pluriel », c'est-à-dire que cette identité scolaire n'est pas exclusive mais va forcément s'articuler - plus ou moins facilement il est vrai - avec des identités familiales, religieuses... qui continuent de caractériser chacun d'entre nous par ailleurs. On peut penser à ce propos que cette conciliation peut être plus difficile pour des enfants « venus d'ailleurs » que pour ceux ayant une longue expérience d'une institution scolaire " en phase » avec la société qui la génère. Autant le mot «intégration » des populations migrantes a pu (et doit) être discuté quand il est associé aux adjectifs, "sociale », "culturelle » etc... autant il devient incontournable quand on parle d'intégration scolaire des jeunes Ena. Ajoutons que cette manifestation d'une identité scolaire est indispensable au sein de l'institution correspondante mais nécessaire aussi dans les marges temporelles de celles-ci, le temps du travail scolaire à la maison par exemple.

\section{Quelques facettes actionnelles de la « compétence scolaire » en classe ordinaire}

En conséquence, nous avons plusieurs fois souligné (cf. Bouchard 2007, Bouchard \& Cortier 2006) qu'il nous semblait urgent de sortir, pour l'accueil de ces publics, d'une conception communicative universaliste de l'enseignement /apprentissage des langues et de viser d'abord à rendre ces élèves capables d'exercer globalement leur « métier » d'élèves au sein de l'institution scolaire française.

Le phénomène sur lequel nous focalisons notre attention, car il nous semble particulièrement important pour les Ena, est celui de leur participation aux interactions scolaires, verbales et non-verbales, en français et «à la française », telles qu'elles se déroulent de fait, quotidiennement, ordinairement, dans nos écoles. Il semble moins « grave » pour un Ena de s'exprimer dans une interlangue que l'enseignant pourra toujours corriger ponctuellement, que de se comporter «interculturellement» d'une manière qui peut apparaître comme non acceptable à un enseignant, amené alors à lui « faire la leçon » publiquement. Ces comportements interactionnels attendus correspondent pour une part aux contraintes fonctionnelles, didactiques et pédagogiques de l'institution scolaire, mais aussi, pour une autre, à des contraintes plus culturelles liées à une tradition éducative et scolaire particulière (en perpétuelle évolution certes mais...), celle de l'école française.

Cette participation exige en particulier une capacité à trouver «sa place » (cf Kerbrat-Orecchioni 1996), et donc à agir « en phase » avec les autres participants pour collaborer à l'action conjointe qui se déroule sous l'impulsion de l'enseignant. Même s'il ne va toujours de soi (cf. le début du corpus ci-dessous et les difficultés de l'engagement dans l'action conjointe), le «phasage » a été appris et renforcé implicitement pour les élèves français par l'expérience partagée d'un temps scolaire vécu - au long cours -. Pour les élèves, plus récemment - brutalement quelquefois - arrivés dans l'institution scolaire française, cette 
coordination reste plus délicate, et cette difficulté varie en fonction de la nature de leur (éventuelle) scolarisation précédente. Ils peuvent avoir pour certains l'expérience d'un vécu scolaire plus « individuel » (les enfants étrangers venant d'Allemagne ou des pays anglo-saxons par exemple) ou au contraire encore plus collectif (ceux venant des "grands groupes» usuels dans les écoles africaines.) Mais cette expérience a toutes chances d'être différente de celle des élèves français dont ils doivent désormais partager le quotidien scolaire.

\subsection{Les interactions verbales}

Une question très classique de compétence scolaire porte ainsi sur le droit à la parole des uns et des autres. Typiquement la classe en tant qu'espace polylogal exige une distribution précise de la parole, évitant les chevauchements et permettant de rendre audibles les dilogues «publics » maître-élève. Il est bien connu - mais peut-être pas encore par tous ces élèves ex-Enaf- qu'une "bonne » réponse n'est pas seulement une réponse juste "scientifiquement » mais aussi un réponse bien formée linguistiquement (cf. interventions métalinguistiques de l'enseignant en caractères gras ci-dessous) et surtout une réponse autorisée interactionnellement et donc respectant le rituel de la demande de parole (cf. Coulon 1993, pour l'analyse ethnométhodologique de décalages interactionnels dans ce moment caractéristique de l'interaction didactique):

\section{Exemple 1 : Corpus Marseille (Cortier-Peutot) :}

1. é : monsieur si

2. En : si tu veux parler tu lèves la main\# ça n'a pas changé... Chérifa

3. é : c c c' est

$\begin{array}{ll}\text { c'est } & \text { avant/ } \\ \text { c'est } & \text { avant/ que l'eau vienne }\end{array}$

4. En : bien ça a poussé avant que l'eau vienne/

ça porte un nom ? /

Chérifa ? ça...ça nous est arrivé à Marseille de temps en temps

5. é : une inondation

6. En : bien... nous avons ici/ affaire/ à une/ inondation

7. é : // je l'ai dit

8. En : $\quad / /$ je sais / tu l'as dit mais j'aimerais que tu lèves la main et que tu aies la parole Simba...

donc ici nous avons un moment/ où le Nil/ est en train de/ le verbe ?

Ces moments d' «interpellation » de l'enseignant par l'élève, sont des moments délicats de l'interaction didactique qui exigent, pour être pertinents, de respecter un rituel communicatif, essentiellement mimogestuel (lever le doigt, se tourner vers l'enseignant...). L'interpellation verbale en "Monsieur» (cf. Détrie 2006) est uniquement tolérée. Ici de plus on voit en lé que ce vocatif est immédiatement suivi par le début de la production d'un énoncé. Ce non-respect du rituel scolaire montre que l'individu-élève ne se conçoit pas comme un simple sujet de l'institution et simultanément refuse donc - de fait - de situer l'interlocuteur à sa «bonne » place institutionnelle. D'où la «remise en place» en 2 Ens, produite par l'enseignant et rappelant l'itérativité de cet incident pédagogique. 
Ces comportements peuvent être liés à des phénomènes interactionnels différents, de nature pédagogique ou didactique : ils peuvent être propres à une discipline ou plus généraux. Certaines disciplines comme l'histoire-géographie ou le français langue «maternelle » par exemple, permettent voire exigent plus de mise en rapport entre individu et sujet scolaire, avec tous les risques d'articulation de cette dualité plus hétérogène sans doute chez l'Ena que chez le jeune natif. Pour le premier plus que pour le second peut se poser alors la question de savoir quand c'est son identité extrascolaire qui tend à s'exprimer en lieu et place de l'identité scolaire, d'élève ou d'apprenant, dont le dote l'activité en cours.

Dans la paire d'exemples 2 et 2', ci-dessous, enregistrés dans des cours d'histoire géographie au collège, on voit l'ambiguïté de l' «interpellation» didactique. Ces exemples mettent en scène non pas des Ena mais des élèves d'origine maghrébine. C'est en tant que tels qu'ils sont dans le premier cas ou qu'ils se sentent dans le second «interpellés » par le maître. Mais on observe qu'ils peinent alors à se définir en même temps comme des sujets de l'institution scolaire française et des individus définis par une histoire personnelle, un lieu de naissance, une sensibilité culturelle :

\section{Exemple 2 :}

$313 \mathrm{P}$ dis-moi nabil est-ce que tu quand l'algérie a est indépendante

quand l'algérie est indépendante

\section{Exemple 2':}

$12 \mathrm{P}$ comment on l'appelle toute la partie d'afrique du nord'

$13 \mathrm{EE}$

$14 \mathrm{P}$ c'était le maghreb

vous vous souv=nez le maghreb on l'avait vu quand on a vu les musulmans 0 bien

\section{fallait $l=$ dire plus fort kamel le maghreb hein 0}

En conséquence on voit que leur parole est chaque fois décalée par rapport à la demande de l'enseignant, à la fois bloquée dans le premier cas, et non complètement assumée dans le second.

De manière significative, l'exemple suivant fait apparaître, chez un Ena cette fois-ci, la réponse inverse au même «double bind", l'identité individuelle l'emporte alors de manière trop marquée sur l'identité scolaire :

\section{Exemple 3 : Corpus thèse Davin $\left(6^{\circ}\right.$ spéciale : français) :}

306. P : pourquoi vous dites c'est une femme'

307. $\mathrm{S}:+$ parce qu'elle pleure

308. P : - il n'y a que les femmes qui pleurent

309. $\mathrm{S}:+$ oui

310. $\mathrm{P}:-\quad$ attendez $+\mathrm{S}$ doit nous donner une explication

311. $\mathrm{S}:+\quad$ ils pleurent parfois mais ils sont forts

P: $\quad$ on reviendra à ce sujet un autre jour+ pourquoi on parle d'un homme

et d'une femme dans ce texte' 


\author{
316. Ni : - les hommes aussi pleurent \\ 317. P : - les hommes aussi pleurent+ \\ vous voyez que Ni réagit après ce que $\mathrm{S}$ a dit \\ 318. $\mathrm{S}:+\quad$ moi je pleure pas \\ 319. P : $\quad$ donc + je disais on a une femme et un homme ++ qu'est ce que vous \\ remarquez à la fin du texte+ moi j'ai pris ma tête dans ma main et j'ai \\ pleuré'
}

Cet exemple montre à la fois l'affirmation «virile» du jeune $\mathrm{S}$ - affirmation liée à ses valeurs extrascolaires - et l'action d'acculturation indirecte de P, l'enseignante, qui « botte en touche » (312) ou s'appuie sur une autre élève (317) pour faire évoluer de manière non autoritaire cette vision du monde. On remarque aussi l'insistance « réflexive » de l'enseignante qui montre la nécessité scolaire d'expliquer, de justifier son point de vue. D'un point de vue argumentatif enfin (cf. Moeschler 1985), on constate que l'élève non natif s'engage dans un échange argumentatif "risqué » avec l'adulte-enseignante en maintenant à quatre reprises sa position. Ce n'est que dans sa quatrième réplique qu'il diminue la portée de son affirmation en passant du général (les hommes) au particulier (moi). Cette fin d'échange montre aussi que c'est bien en tant qu'individu et non pas en tant que sujet de l'institution scolaire que S. en est venu à s'exprimer à l'occasion de cette explication de texte. On peut penser aussi que cette séquence, ressentie comme moins contrainte institutionnellement et didactiquement, amène $\mathrm{S}$. à tester publiquement le rapport maître-élève à la française, voire au-delà le rapport de genre tel qu'il se peut licitement se manifester dans l'enceinte de la classe.

L'Ena doit donc se familiariser avec les activités langagières telles qu'implicitement elles peuvent/doivent se dérouler à l'école, en fonction des droits et devoirs de parole des différents partenaires de l'interaction complémentaire, inégale qui s'y joue. Mais il doit plus rapidement encore s'initier aux pratiques non langagières, typiques de l'école (française). Ce sont même celles-là qu'il importe de travailler en priorité avec lui, afin qu'il puisse le plus rapidement possible être socialisé « en actes » dans la classe qui correspond à son age et à son cursus normal. C'est de la qualité de cette socialisation que va dépendre la poursuite de son apprentissage du français comme des autres disciplines

\title{
3.2 Les inter-actions pédagogiques
}

Nous avons l'habitude de préciser cette dualité verbale et non-verbale des interactions pédagogiques de deux manières. D'une part en «parlant» d'inter-actions et non pas d'interactions. D'autre part en les qualifiant à l'aide de deux adjectifs un peu barbares certes, mais qui ont l'avantage d'être explicites : elles sont à la fois pluripraxiques et oralographiques (cf Bouchard à paraître). Nous voulons dire par là que les élèves doivent apprendre à agir collectivement et de manière coordonnée au rythme imprimé par l'enseignant (polypraxie) et que, parmi ces actions, beaucoup ont trait au maniement simultané de la langue orale et des outils d'écriture (oralographisme). C'est cette coordination des actions du maître et des élèves qui leur permet de rester en phase de manière à ce que le groupe puisse progresser à la fois individuellement et collectivement.

Certes ces obligations ne différent pas pour les élèves étrangers et pour les élèves français et les infractions à la règle correspondantes non plus. Les classes dites "difficiles » sont des classes précisément où n'existe pas ce consensus scolaire, ce partage d'une même compétence scolaire, que celleci n'ait pas pu être construite ou que celle-ci se heurte à un refus d'élèves qui se veulent ascolaires. Mais il n'empêche que le freinage de l'action collective consécutif à ces déphasages, semble assez caractéristique des classes scolairement pluriculturelles. Le problème pour ces élèves, néo-arrivants, « allopraxiques » (si on forge ce néologisme sur le néologisme déjà existant " alloglottes ») est - avant d'adhérer ou de refuser cette culture scolaire - de découvrir, par essai et erreur, le comportement collectif acceptable dans ce nouveau milieu. 
Cet exemple montrait les conséquences purement verbales, un échange argumentatif long entre enseignante et Ena, d'un incident bénin d'origine interculturel. Le décalage des habitus scolaires peut avoir aussi des manifestations mixtes, à la fois verbales et non-verbales comme le montre l'exemple 2 cidessous :

\section{Exemple 4 : Corpus Cortier-Peutot : classe de dispositif Marseille $6^{\circ}$ :}

9.

Ens : Maintenant
Fatma tu parles trop.

on va

se pencher sur cette image/

on va se pencher un/

se pencher un peu sur cette image/

bon/ on se calme/ Mohamed... d'accord?

c'est pas de::: des beaux arts que je demande hein...c'est bon ?...

Fatima ? qu'est-ce qui te manque ? ça c'est pas grave... regarde/ tu prends ton euh (28') voi ::: là tu t'arrêtes là... c'est fini... d'accord ?

ça tu gommeras chez toi/ mets ta gomme ici...

BON...

qu'est que je vois?

est-ce que je vois tout... d'un seul coup ?
10. E l: (bavardages)

11. Ens : chut :::

qu'est-ce que je vois? OUI\#

Dans cet exemple enregistré dans une classe d'Histoire-Géographie «spéciale » regroupant des Enaf, on constate que la production de la consigne qui va ouvrir l'épisode de commentaire d'un document est retardée par plusieurs interactions parasites. Il s'agit d'une part d'interactions « disciplinaires », visant le comportement locutoire (Fatma) ou actionnel (Mohamed) d'élèves sortant de la marge de tolérance acceptée par l'enseignant, représentant des us et coutumes scolaires à la française. Mais il s'agit aussi (cas de Fatima), de comportements et d'actions, scolairement acceptables, mais décalés temporellement par rapport à l'avancée du temps didactique que veut créer l'enseignant. Le problème ressort plus à l'organisation du travail et de sa répartition entre travail public - en classe - et travail privé - à la maison. Remarquons qu'il s'agit aussi de la réalisation, parallèlement à l'interaction orale d'un croquis de géographie. Il s'agit donc d'un travail d' «inscription» sollicitant une utilisation experte d'outils graphiques et le respect d'une symbolisation iconique spécifique, caractéristique d'une conception littéraciée de l'apprentissage. Cet exemple illustre bien la diversité des engagements dans l'action conjointe de ses jeunes apprenants non natifs souligné par V. Rivière (2005).

Les diverses «remises en place» rendues nécessaires ont aussi pour conséquence de couper et de complexifier l'énonciation de la consigne par l'enseignant, amené à la répéter et à la reformuler au détriment des élèves les plus fragiles linguistiquement.

En définitive donc cette compétence scolaire s'illustre par des phénomènes liés soit à l'activité individuelle, avec les prises de parole et les risques propres que prennent leurs auteurs soit à l'activité collective, guidée par l'enseignant, et les problèmes de mise en phase qui peuvent se poser.

Dans un premier temps c'est surtout à cette seconde réalité que va être confronté l'Ena qui avant d'être un locuteur actif devra être un participant actif. Pour l'être effectivement il faudra donc qu'il développe une 
compréhension suffisante de la parole de l'enseignant, et en particulier des tours de parole opérant des transition entre les différentes « transactions » (Roulet 1985) qui vont se succéder au cours de la leçon.

\section{Exemple 5 : Corpus mathématiques primaire 80 :}

$1 \mathrm{M}$

croisez les bras

regardez

au tableau bien

deux fois un égale combien

$27 \mathrm{M}$ bien

bien alors

regardez bien

au tableau

246820243228 ce sont des multiples de deux

$47 \mathrm{M}$ bien

regardez ce nombre

regardez bien ensemble au tableau

Dans cet exemple, il est vrai un peu daté, on remarque qu'à vingt tours de parole de distance, l'enseignant utilise la même procédure pour opérer une transition entre une première, une seconde puis une troisième transaction didactique. Il utilise comme marqueur de fin de la transaction en cours une marque évaluative positive à rôle de bornage. Il ouvre la nouvelle transaction sur un mode oralographique, en la réancrant sur une inscription figurant au tableau. ). Pour ce faire, il utilise des expressions parallèles, dont la proximité formelle indique par leur forme même la similitude des transitions ainsi signalées.

Le rôle de l'écrit dans cette organisation de l'interaction longue est bien sûr en lui-même intéressant à souligner : la classe est un espace ou l'écrit quand il n'est pas explicitement dominant est toujours sousjacent à l'inter-action en cours. Il est donc nécessaire aux Ena d'avoir rapidement une maîtrise de cet écrit du tableau noir en particulier. Remarquons cependant qu'il s'agit plus d'un écrit du mot que d'un écrit du texte (cf. Bouchard à paraître). Mais insistons sur le fait que cette maîtrise du mot et des listes de mot est un acquis culturel essentiel pour l'individu comme pour les civilisations (cf Goody).

Ces prises de parole stratégiques de l'enseignant que nous venons d'évoquuer à la frontière des 《 transactions » internes à l' « incursion » pédagogique pour reprendre les termes de Roulet, jouent un rôle encore plus déterminant lors de l'ouverture de ces incursions. C'est le cas en particulier, dans le primaire, pour l'ouverture globale de la journée par le maître qui est responsable non seulement de l'ensemble des disciplines mais aussi de l'habitus scolaire global de sa classe. 


\section{Le début de cours comme moment stratégique de l'interaction polypraxique}

\section{1}

Le court extrait qui suit, enregistré dans un CM1, montre ainsi la mise en place d'une classe au commencement de la journée. Il fait apparaître la diversité des actions auxquelles doivent se livrer enseignant et élèves, et la routine qu'elles représentent.

Exemple 6 (classe de CM1- Ecole primaire - Bourg - 2007) :

\begin{tabular}{|c|c|}
\hline 5 Discours oral & 5.1.1.1 Gestualité - situation \\
\hline $\begin{array}{l}\text { 1P : allez entrez // on va essayer } \\
\text { de démarrer rapidement }\end{array}$ & $\begin{array}{l}\text { - les élèves entrent derrière } \\
\text { l'enseignante, se rendent à leur place } \\
\text { respective - brouhaha - } \\
\text { plusieurs élèves ressortent dans le couloir pour } \\
\text { s'inscrire sur une liste et reviennent un moment plus } \\
\text { tard dans la classe }\end{array}$ \\
\hline \begin{tabular}{llr} 
1.1.P : y a quelques & \multicolumn{2}{c}{ a } \\
autorisations \\
manquaient / c'est bon
\end{tabular} & regarde une liste - un élève donne un document \\
\hline & $\begin{array}{l}\text { attend que tous les élèves reviennent dans la } \\
\text { classe }\end{array}$ \\
\hline 1.2.P : tout le monde est là' & se dirige vers la porte et la ferme \\
\hline \multicolumn{2}{|c|}{ (...) présente brièvement les 2 collègues qui sont là pour les besoins du film } \\
\hline $\begin{array}{l}\text { 2P : on va commencer plutôt par } \\
\text { / quelques / documents que } \\
\text { je vous avais demandé de } \\
\text { faire signer par vos parents / } \\
\text { alors pour aller un petit peu } \\
\text { vite / il y avait euh rappelez- } \\
\text { moi } \\
\text { 3E1 : la poésie et } \\
\text { 4P : poésie' non / } \\
\text { 5E 2: mais non } \\
\text { 6E 3: non la fiche de lecture et } \\
\text { l'évaluation d'histoire / } \\
\text { 7P : alors ce que je propose } \\
\text { c'est que / Elise tu récupères } \\
\text { les évaluations d'histoire s'il }\end{array}$ & \\
\hline
\end{tabular}




\begin{tabular}{|c|c|}
\hline $\begin{array}{l}\text { te plait // } \\
8 \mathrm{E} 4 \text { : les cahiers } \\
\text { 9P : et puis euh / Lorena tu } \\
\text { ramasses les cahiers pour la } \\
\text { fiche de lecture / rapidement }\end{array}$ & \\
\hline & $\begin{array}{l}\text { les élèves ouvrent leurs classeurs pour y prendre } \\
\text { les documents demandés }\end{array}$ \\
\hline $\begin{array}{l}\text { 9.1.P : pour ceux qui ont oublié } \\
\text { on règlera ça en début } \\
\text { d'après-midi // s'il y a des } \\
\text { oublis }\end{array}$ & \\
\hline & $\begin{array}{l}\text { l'enseignante ouvre le tableau, regarde un } \\
\text { moment les élèves en train de rendre les } \\
\text { documents } \\
\text { sur le tableau est écrit: }\end{array}$ \\
\hline & $\begin{array}{l}\text { Le compte est bon } \\
\text { Réservoir des nombres : } 24-8-10-250\end{array}$ \\
\hline 9.2.P : les cahiers' & $\begin{array}{l}\text { cherche du regard où les faire poser, se tourne } \\
\text { vers le bureau à sa gauche et libère un peu de } \\
\text { place dessus } \\
\text { regarde les élèves en train de rendre les } \\
\text { documents }\end{array}$ \\
\hline $\begin{array}{l}\text { 9.3.P : alors pendant qu'on } \\
\text { termine // de ramasser les } \\
\text { documents // on va démarrer } \\
\text { ce matin par une séance de } \\
\text { calcul rapide en reprenant / } \\
\text { un exercice que je vous avais } \\
\text { donné la semaine dernière } \\
\text { hein le compte est bon / } \\
\text { donc je vais vous rendre les } \\
\text { fiches / et comme c'est un } \\
\text { petit peu difficile on va } \\
\text { revoir / les règles // }\end{array}$ & \\
\hline & regarde les élèves qui ramassent les documents \\
\hline $\begin{array}{l}\text { 9.4.P : et essayer de trouver } \\
\text { quelques méthodes pour euh } \\
\text { être efficace }\end{array}$ & \\
\hline $\begin{array}{l}\text { 9.5.P : c'est bon' tout est } \\
\text { ramassé merci ça je vais le } \\
\text { poser là }\end{array}$ & $\begin{array}{l}\text { prend un paquet de feuilles que lui tend Elise et } \\
\text { les pose sur un bureau à sa droite }\end{array}$ \\
\hline $\begin{array}{l}\text { 9.6.P : bon vous avez tout } \\
\text { ramassé merci ça je vais le poser }\end{array}$ & $\begin{array}{l}\text { montre du doigt à Lorena le bureau de gauche où } \\
\text { poser les cahiers }\end{array}$ \\
\hline
\end{tabular}




\begin{tabular}{|l|l|}
\hline $\begin{array}{l}\text { là et Lorena les cahiers tu les } \\
\text { poseras juste // de ce côté là voilà }\end{array}$ & montre le tableau du doigt \\
\hline 9.7.P : voilà / alors // & $\begin{array}{l}\text { rappelle à l'ordre les élèves qui ne regardent pas } \\
\text { encore le tableau - toujours en montrant du doigt } \\
\text { le tableau }\end{array}$ \\
\hline 9.8.P : c'est de ce côté & se tourne vers le tableau \\
\hline $\begin{array}{c}\text { 9.9.P : donc vous vous souvenez } \\
(\ldots)\end{array}$ & \\
\hline
\end{tabular}

Certaines habitudes sont purement praxiques : attendre dans le couloir que l'enseignant donne l'ordre de rentrer, se diriger vers sa place habituelle et non vers une place choisie à chaque entrée dans la classe. Celles-ci sont systématiques, totalement routinières, et ne font donc l'objet d'aucune expression langagière, sauf en cas de transgression. D'autres ne nécessitent que quelques mots - très elliptiques pour une « oreille extérieure » vous pensez à la cantine, y a quelques autorisations // qui manquaient - pour se déclencher. Et même lorsque la consigne est plus explicite, pour le ramassage des cahiers, par exemple, (tours de parole 7 et 9), la rapidité avec laquelle les élèves la mettent en œuvre montre qu'il s'agit également d'une habitude bien établie. D'autres règles concernent l'occupation de l'espace : en rentrant dans la classe, pendant l'inscription à la cantine, pendant que l'on ramasse les devoirs, les élèves peuvent se déplacer, rester debout, bavarder entre eux. A partir du moment où la leçon proprement dite commence, le comportement devient collectif, il faut s'asseoir, se taire, regarder ensemble le tableau, écouter en silence l'enseignante (tour 9.8). Outre ces routines actionnelles, apparaissent les routines communicatives telles que l'annonce par l'enseignante du déroulement de la séance on va démarrer ce matin par une séance de calcul rapide ... (tour 9.3 ), annonce généralement reliée au travail de la séance précédente.

Cet habitus scolaire dont l'assimilation participe largement à la compétence scolaire, comme on l'a dit plus haut, exige une adaptation permanente dans la mesure où il se décline diversement selon les cours et les enseignants. La politesse est de règle pour tous mais tel enseignant demande à chaque élève de dire bonjour en entrant dans la classe (tu pourrais répondre dit cette enseignante à un élève qui ne réagit pas à son «bonjour») (Cours de mathématiques, collège de Bourg 2007), alors que d'autres se contentent d'une salutation plus collective. Le ramassage des travaux est récurrent mais certains le font faire par un élève quand d'autres le font eux-mêmes, ou encore demandent aux élèves de les poser sur le bureau en arrivant. Le respect de l'espace-enseignant n'est pas le même partout: s'il est clair qu'aucun élève ne s'installe jamais au bureau de l'enseignant, passer derrière le bureau de l'enseignant pour rejoindre sa place peut être diversement interprété : indifférence pour les uns, interdiction pour les autres (pas derrière le bureau // je l'dis à chaque fois) (ibidem). Chaque enseignant investit de manière diversifiée un schéma global et commun de fonctionnement de la classe.

La classe est donc un lieu d'habitudes - qui, du point de vue des acteurs intégrés, sont même de véritables évidences - que l'élève arrivant doit rapidement assimiler pour avoir un comportement considéré comme normal.

A ces habitudes comportementales, s'ajoute bien évidemment la maîtrise de la communication verbale scolaire dont la complexité est à la mesure de la diversité des rôles que joue l'enseignant face à ses élèves et de tout ce qui se joue simultanément dans une classe. L'extrait ci-dessous, début d'un cours de chimie en 5e permet de mesurer cette complexité actionnelle et discursive. 


\section{Exemple 7 : $\quad$ Cours de physique : L'eau dans l'environnement}

Classe de $5^{\mathrm{e}}$ (avril 2004 - collège Lyon)

1 Pallez vous commencez par vous installer vous taire vous calmer // (plusieurs secondes)

vous sortez vos cahiers de texte pour la semaine prochaine et vous vous taisez // on est d'accord //

$2 \mathrm{E}(\ldots)$

3 Ppardon / on va en parler après de ça / pour l'instant je vous demande qu'une chose / première chose se taire deuxième chose / donc $\mathrm{y}$ a deux choses / à faire tout de suite c'est se taire on va mettre pas trois heures à commencer et sortir son cahier de texte et noter que la prochaine semaine dans la prochaine semaine qu'est ce que je voulais vous dire

4 E29 avril (?)

5 Pdonc on sera le

6 E 29 avril

7 P 29 avril // donc vous marquez pour le 29 / avril contrôle / contrôle sur le chapitre 1 et 2 de la chimie alors c'est bien d'accord ce sera plutôt sur le chapitre 2 mais le chapitre 1 y a y a toutes les bases donc faut sa faut toujours savoir son cours //

donc on est le / 22 / alors qui c'est qui est 14812162024 // 24 vous êtes 24 tout le monde est là //

$$
8 \mathrm{EE} \quad \text { (inaudible) }
$$

9 P après on en parle allez on commence le cours // (plusieurs secondes)

je crois que j'ai du mal à être clair là ce matin hein Yasmina j'ai dit quoi / j'ai dit on commence on se tait donc je crois que ça fait un moment que je l'ai dit ça / alors si vous vous rappelez bien ce qu'on a fait donc avant les vacances on a vu / comment / reconnaître / la présence / ou non / d'eau / dans les aliments / dans les boissons / donc on a vu / dans les boissons y avait toujours de l'eau / que dans les aliments / c'était / variable / la plupart ont de l'eau / à en plus ou moins grande / quantité // certains n'ont / pas du tout d'eau / c'est qui c'est qui qui a pas d'eau

10 E l'huile

$11 \mathrm{P}$ l'huile d'accord / certains en ont très peu exemple la semoule les lentilles qu'on avait fait // d'accord / donc aujourd'hui pour finir ce cours c'est moi qui vais faire rapidement l'expérience c'est la même que celle qu'on a faite je voudrais qu'on a qu'on aille assez vite on va regarder ce qui se passe par rapport / à des produits qu'on utilise à la maison c'est-à-dire qu'on va regarder est-ce qu'il y a ou non de l'eau avec des produits / qu'on utilise / à la maison alors vous avez vu je vous ai fait une feuille eau écarlate / white spirit / liquide vaisselle incolore lessive / liquide // antiseptique / et sirop / incolore / donc on va en tester seulement 2 / que l'on a / les autres on les a pas puis les autres on va en reparler / c'est assez compliqué.

(...)

$\mathrm{X} \mathrm{P}$ alors le white spirit // on va tester / alors je vais essayer d'en faire tomber très peu là c'est un peu compliqué euh comme il m'a pas tout amené euh j'ai j'ai au moins ça je vais faire tomber une goutte / en utilisant / allez vous vous taisez parce que ça commence à m'énerver là / y en a déjà qui ont été punis la dernière fois pour les mêmes raisons / alors je vais faire tomber une goutte sur le tas / vous voyez la technique hein 
qu'on qu'on peut utiliser quand on a pas forcément euh de compte goutte on peut utiliser un agitateur et ça fait pareil alors j'ai fait tomber / donc du white spirit sur / un des tas alors pour l'instant vous voyez pas grand chose je vais vous montrer // donc c'est celui qui est à gauche / pour vous / donc euh pour vous c'est à droite alors celui qui est à droite est-ce qu'il a changé de couleur // alors je vais vous faire la même chose cette fois-ci vous allez voir la différence parce que je crois que vous avez un peu / dormi pendant les vacances avec le liquide vaisselle alors le liquide vaisselle c'est celui qui est pour vous à gauche et le white spirit à / droite alors regardez / et vous me dites / donc vous en tirez une conclusion alors white spirit je rappelle c'est à droite et liquide vaisselle à gauche // donc vous regardez ce que vous a.. vous observez et on va en parler tout de suite // c'est bon tout le monde voit // (plusieurs secondes) tout le monde a tout le monde a vu

L'enseignant est à la fois expert d'une discipline (la chimie), organisateur d'activités d'apprentissage (observer des réactions chimiques pour détecter la présence d'eau dans les produits quotidiens), régulateur de comportements (obtenir le silence), gestionnaire de la classe (vérifier les présences), etc. La concomitance de ces rôles ainsi que le poids de la situation et des actions qui s'y déroulent impliquent une construction discursive souvent complexe de la parole enseignante avec des énoncés de différents niveaux fortement imbriqués les uns dans les autres. L'élève allophone doit développer rapidement la compétence langagière qui lui permettra de comprendre et de participer à ces échanges.

\section{L'élaboration d'outils pédagogiques spécifiques à partir d'enregistrements de classe ordinaires}

La solution de ces problèmes d'action scolaire, verbale et non verbale, passe alors par deux opérations qui ressortissent autant de l'ingénierie éducative que de la didactique des langues à proprement parler. Il s'agit d'une part de la création de dispositifs de formation permettant d'articuler pédagogiquement mais aussi administrativement (horaires, ouverture des classes, circulation des Ena...) classes d'accueil et classes-cibles «normales », c'est-à-dire - et c'est de plus en plus communément admis - de viser une intervention « éducative » qui aille au delà d'une simple action linguistique dans une classe d'accueil qui risque toujours de se refermer sur elle-même et de se trouver isolée même si elle utilise la méthodologie la plus adaptée à ces publics d'Ena.

Il s'agit d'autre part de nourrir cette mise en relation par l'élaboration d'un matériel hybride, tenant aussi bien de la classe de FLS que de la classe cible de chimie par exemple, en repérant et en isolant des microsituations-clés de cette vie de classe, pour, après les avoir enregistrées en vidéo, les avoir transcrites et analysées, les didactiser en tentant de les rendre audibles et visibles hors contexte, de manière autonome, en les simplifiant, les fictionnalisant le cas échéant... comme on le fait dans le cadre en général du FOS (cf. Mangiante et Parpette 2005).

C'est pourquoi se mettent en place des équipes regroupant enseignants de français langue seconde, enseignants d'autres disciplines et chercheurs en didactique des langues. Leur but est d'étudier les possibilités de recourir à des enregistrements de classes ordinaires comme supports pédagogiques pour l'apprentissage du français de solarisation et, plus largement, des compétences scolaires.

Sur le plan didactique, la démarche s'inscrit dans la problématique des situations et documents authentiques, qui repose sur l'hypothèse qu'une confrontation avec les situations cibles favorise l'apprentissage. Cette hypothèse s'impose d'autant plus pour les discours oraux que leur réalité est mal connue et, de ce fait, leur complexité largement sous-évaluée.

Les premiers travaux réalisés au sein du laboratoire ICAR sur cette question (Parpette \& Peutot 2006) ont commencé à mettre à jour à la fois l'intérêt mais aussi les contraintes de la transformation de ces corpus de classes en supports d'apprentissage. 


\subsection{Le traitement de la dimension actionnelle}

Le cadre d'un article ne permet pas de passer en revue les nombreuses possibilités de traitement didactique des situations scolaires auprès des élèves allophones en cours d'apprentissage des compétences scolaires. Nous nous limiterons aux aspects mis en évidence par les extraits des exemples 6 et 7 présentés plus haut.

A partir de l'exemple 6, trois aspects peuvent être abordés, à travers un questionnement des élèves devant des extraits vidéos de classe :

- L'entrée en classe : qui entre en classe le premier, les élèves ou le professeur? où s'installent les élèves, à une place attitrée ou librement? le maitre (professeur) et les élèves disent-ils bonjour? que fait un élève qui arrive en retard ?

- Les différentes actions : à quel moment commence la nouvelle leçon ? que se passe-t-il avant? qui ramasse les copies, le maitre (professeur) ou un élève ? la classe est-elle silencieuse ? à partir de quand les élèves doivent-ils se taire ?

Le début de la nouvelle leçon: comment voit-on que la nouvelle leçon commence ? sur quoi s'appuie la leçon, sur le tableau? sur le livre ? sur un document donné par le maître (professeur)?

Si l'on regarde l'extrait de cours de physique, on voit que ces questions gardent leur pertinence, signe de l'existence d'un modèle global, stabilisé, de déroulement de la classe et, en l'occurrence, de début de cours. L'entrée en classe se fait dans un brouhaha admis le temps que les élèves s'installent; le début de la leçon proprement dite est précédé d'actions de gestion pédagogique (l'inscription sur le cahier de textes du contrôle de la semaine suivante, la vérification des présences); l'enseignant instaure ou tente d'instaurer le silence; enfin arrive la présentation de la nouvelle leçon, explicitement inscrite par l'enseignant dans la continuité du cours précédent. L'analyse par les élèves allophones d'une série de débuts de classe doit les amener à construire progressivement à travers des exemples locaux un modèle global du fonctionnement de la classe et d'en intégrer différente déclinaisons. Selon le passé scolaire des élèves et le degré de proximité des systèmes éducatifs, cette construction cognitive de la dimension actionnelle de la classe demandera une adaptation plus ou moins importante, et se réalisera donc plus ou moins rapidement.

\subsection{Le traitement de la dimension discursive}

Des actions aux discours, on peut envisager un traitement en continuum. Après l'étape de réflexion sur les déplacements, les mouvements, les objets, la suivante peut consister à demander aux apprenants allophones de mettre en relation les actions des élèves avec les paroles de l'enseignant. Ainsi pour le corpus 6 :

Que dit l'enseignant qui entraîne les actions suivantes?

des élèves ressortent dans le couloir (vous pensez à la cantine)

la maîtresse va fermer la porte (tout le monde est là')

les élèves ouvrent leur classeur et prennent une copie (Elise tu récupères les évaluations d'histoire)

une élève commence à ramasser les cahiers (Lorena tu ramasses les cahiers)

Un travail du même ordre peut être fait à partir de situations telles que celle que montre le corpus 7, dans lequel l'enseignant réalise une expérience devant les élèves en la commentant au fur et à mesure (cf. XP de alors le white spirit... à vous observez et on va en parler tout de suite). Un travail de compréhension peut ainsi être mené à travers la mise en correspondance entre ce qui apparaît à l'image et ce que dit l'enseignant - vous voyez (...) on peut utiliser un agitateur, $j$ 'ai fait tomber du white spirit sur un des tas, le liquide vaisselle c'est celui qui est pour vous à gauche, etc, - puis en demandant aux élèves de reformuler l'expérience à partir de l'image. 
Dans une troisième étape, les passages plus verbo-centrés peuvent être traités par un questionnement sur le contenu linguistique des énoncés (exemple 5):

sur quoi porte la leçon? (calcul rapide)

ce n'est pas une nouvelle leçon: que dit la maîtresse qui le montre ? (reprendre un exercice donné la semaine dernière)

pourquoi reprend-elle cette leçon? (c'est un petit peu difficile).

Ce travail de compréhension peut s'accompagner d'un exercice linguistique sur le futur périphrastique, toujours très présent dans ce type de discours.

Nous avons souligné plus haut la complexité discursive liée à l'imbrication des actes de parole du discours de l'enseignant. Nous avons déjà eu l'occasion d'évoquer le traitement de cet aspect discursif dans la formation linguistique des élèves allophones (Parpette \& Peutot 2006). Dans un premier temps, afin de sensibiliser progressivement les élèves à cette construction, la stratégie peut consister à traiter l'input pour isoler dans une première étape l'énoncé explicatif, en le débarrassant des énoncés latéraux (précisions, ou régulation de la discipline). Cette première phase aboutit à un discours réduit, tel que...

\section{Exemple 8}

alors le white spirit // on va tester / alors je vais essayer d'en faire tomber très peu là (...) je vais faire tomber une goutte / (...) sur le tas / (..) alors j'ai fait tomber / donc du white spirit sur / un des tas alors pour l'instant vous voyez pas grand chose je vais vous montrer // donc c'est celui qui est à gauche / pour vous / donc euh pour vous c'est à droite alors celui qui est à droite est-ce qu'il a changé de couleur // etc..

... après suppression des énoncés :

- allez vous vous taisez parce que ça commence à m'énerver là / y en a déjà qui ont été punis la dernière fois pour les mêmes raisons /

- vous voyez la technique hein qu'on qu'on peut utiliser quand on a pas forcément euh de compte goutte on peut utiliser un agitateur et ça fait pareil

- etc.

Dans une seconde phase, ces derniers sont restitués. Le travail de compréhension orale est ainsi mené en deux temps, ce qui permet aux élèves de s'habituer progressivement à ce ces inputs complexes, sans être noyés sous la difficulté d'une confrontation immédiate avec ce type de discours.

\section{Discours authentiques vs. discours reconstitués, fictionnalisation...}

Reste la question des supports sur lesquels appuyer ces séances de réflexion sur le déroulement de la classe française. Le travail direct sur enregistrements vidéo de classes, s'il est dans son principe intéressant par le rapprochement qu'il constitue avec les situations réelles, pose très souvent des problèmes de compréhensibilité pour un regard extérieur (Parpette \& Peutot ibidem). Le changement de contexte communicatif - l'élève qui regarde un enregistrement de classe n'est pas membre de cette classe et en ignore le vécu - limite les possibilités de recours aux enregistrements de classes authentiques. $\mathrm{Si}$ ceux-ci offrent des possibilités intéressantes dans certains cas, la constitution de supports destinés à des niveaux peu avancés a tout à gagner à envisager également des simulations de cours en veillant naturellement à ce qu'elles intègrent les phénomènes discursifs analysés sur les discours authentiques. Les concepteurs disposeront alors de la latitude que donnent les reconstitutions, en particulier en matière de progression (Bouchard, Cortier, Parpette, à paraître). 
Ajoutons que cette approche de la classe française par le biais de supports pédagogiques s'attachant à la représenter, gagnerait à être combinée - comme, seule, le permet la mise sur pied de dispositifs - à des observations de classes ordinaires, accompagnées par le professeur de la classe d'accueil. Celles-ci complètent et mettent à l'épreuve la compréhension des enregistrements des comportements et des discours des classes ordinaires. Elles constituent une étape importante entre l' « isolement » dans la classe d'accueil et l'immersion dans la classe-cible.

\section{Conclusion}

C'est donc le travail simultané sur ces deux chantiers qu'il nous emble nécessaire de poursuivre, afin de consolider l'action didactique en classe d'accueil en l'appuyant sur une meilleure connaissance du fonctionnement interactionnel de l'enseignement « en français » des diverses disciplines dans les écoles et collèges français. Les différents renforcements, évoqués ci-dessus, de l'apprentissage guidé par la prise en compte des potentialités d'acquisitions sociales offertes par l'environnement scolaire sont d'autant plus importantes à prendre en compte qu'ils correspondent à notre double possibilité d'intervention didactique (classe d'accueil) et adidactique (environnement de la classe d'accueil) effective au sein de l'institution éducative.

Ajoutons que ce dédoublement de la réflexion et de l'intervention didactiques n'est pas spécifique à la problématique de la formation langagière des Ena mais caractérise une époque de globalisation qui modifie le marché des langues. Celle-ci les rend plus fréquemment, plus facilement disponibles hors de la classe de langue, et tend à transformer l'enseignement des langues étrangères en un enseignement, peu ou prou, de langues devenant secondes, qui vise alors à combiner apprentissage guidé et acquisition sociale. Cette combinaison peut s'opérer plus facilement lorsque l'enseignement-apprentissage s'opère comme pour les Ena en situation homoglotte (cf. méthode « Ici ») par la prise en compte des divers entours des classes de langue. En situation hétéroglotte cet appui sur des ressources langagières non didactiques, authentiques, est maintenant rendu possible grâce aux Tic et à internet.

\section{Bibliographie}

Abry, D. Fert, C. Parpette, C. Stauber, J. (2007) ICI, méthode de français, Cle international

Bertrand, Viala, Vigner, G. (2000), Français langue seconde, CNDP

Bouchard, R. (2008), Du français fondamental à la compétence scolaire en passant par le français de scolarisation, Le Français dans le monde : recherches et applications

Bouchard, R. \& Cortier, C. (2005), Français de scolarisation » et mathématiques (comme exemple de culture scolaire-disciplinaire) : une problématique pour les dispositifs d'intégration des Enav,? » in Delefosse, J. M. O. (ed.) Acquisition, Pratiques langagières, Interactions et Contact, Cdrom, Paris 3

Bouchard R. (à paraître) La compétence scolaire comme compétence oralographique : une cible décisive pour l'enseignement du français langue seconde aux enfants nouvellement arrivés en France, Diversité 153, Inrp

Bouchard, R. Cortier, C. (2007), L'intégration scolaire des enfants étrangers : quelle responsabilité didactique et éducative pour la linguistique appliquée ? Français de scolarisation et compétence scolaire en histoire/géographie, Bulletin Suisse de Linguistique Appliquée, Neuchâtel

Bouchard, R. Parpette, C. Cortier, C. (à paraître 2008) L'appropriation du français par les élèves nouveaux arrivants dans les dispositifs d'intégration, Le Français dans le monde : recherches et applications

Cortier, C. (2003), FLE/FLS/FLM ? Quelles répartitions et quelles transitions pour la scolarisation des élèves allophones et leur intégration dans les classes ordinaires du cursus français? (Textes officiels et pratiques scolaires), in Defays, J.M., Delcominette, B., Dumortier, J.L., Vincent, L. (dir.), (2003), L'enseignement du français aux non-francophones. Le poids des situations et des politiques linguistiques, E.M.E., Fernelmont

Davin-Chnane, F. (2005), Didactique du français langue seconde en France. Le cas de la discipline " français » au collège, Thèse de doctorat, Université de Provence, Aix en Provence 
Détrie, C. (2006) De la non-personne à la personne : l'apostrophe nominale, Paris CNRS.

Kerbrat-Orecchioni, C. (1996), La conversation, Seuil, Paris

Lahire, B. (2001), L'homme pluriel, les ressorts de l'action, Hachette.

Martinez, P. (2002) (dir.), Le français langue seconde; apprentissage et curriculum, Maisonneuve-Larose, Paris, $101-131$

Parpette, C. (2005), Réflexions sur la parole enseignante en français langue seconde », Actes du séminaire interrégional de recherche-action, Ecole Normale Supérieure de Nha Trang, Nha Trang, Vietnam

Parpette, C. (2008), «Les discours pédagogiques oraux : évolution des représentations et des pratiques didactiques », Le français dans le monde, Recherche \& Applications 43, Cle International, Paris

Parpette, C. Peutot, F., (2006), «Les enregistrements de classe ordinaires comme support d'enseignement du français langue de scolarisation », Cahiers de l'ACEDLE 2, revue en ligne, Paris, 165-177

Pochard, J.-C. (2003), Le français langue seconde hôte, un cas limite de français langue seconde,

Porcher, L. (1978), La scolarisation des enfants étrangers en France, Didier-CREDIF, Paris

Verdelhan-Bourgade, M. (2002), Le français de scolarisation. Pour une didactique réaliste, PUF, Éducation et formation, Paris

Vigner, G. (2001), Enseigner le français comme langue seconde, Clé international, Paris 